Annales Geophysicae (2003) 21: 709-717 (C) European Geosciences Union 2003

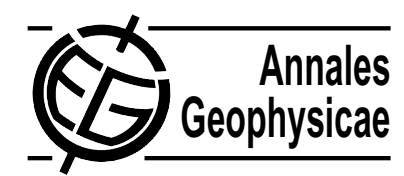

\title{
April 2000 geomagnetic storm: ionospheric drivers of large geomagnetically induced currents
}

\author{
A. Pulkkinen ${ }^{1}$, A. Thomson ${ }^{2}$, E. Clarke ${ }^{2}$, and A. McKay ${ }^{3}$ \\ ${ }^{1}$ Finnish Meteorological Institute, Geophysical Research Division, P.O. Box 503, FIN-00101, Finland \\ ${ }^{2}$ British Geological Survey, Edinburgh, EH9 3LA, UK \\ ${ }^{3}$ Department of Geology and Geophysics, University of Edinburgh, EH9 3JW, UK
}

Received: 15 May 2002 - Revised: 15 August 2002 - Accepted: 30 August 2002

\begin{abstract}
Geomagnetically induced currents (GIC) flowing in technological systems on the ground are a direct manifestation of space weather. Due to the proximity of very dynamic ionospheric current systems, GIC are of special interest at high latitudes, where they have been known to cause problems, for example, for normal operation of power transmission systems and buried pipelines. The basic physics underlying GIC, i.e. the magnetosphere - ionosphere interaction and electromagnetic induction in the ground, is already quite well known. However, no detailed study of the drivers of GIC has been carried out and little is known about the relative importance of different types of ionospheric current systems in terms of large GIC. In this study, the geomagnetic storm of 6-7 April 2000 is investigated. During this event, large GIC were measured in technological systems, both in Finland and in Great Britain. Therefore, this provides a basis for a detailed GIC study over a relatively large regional scale. By using GIC data and corresponding geomagnetic data from north European magnetometer networks, the ionospheric drivers of large GIC during the event were identified and analysed. Although most of the peak GIC during the storm were clearly related to substorm intensifications, there were no common characteristics discernible in substorm behaviour that could be associated with all the GIC peaks. For example, both very localized ionospheric currents structures, as well as relatively large-scale propagating structures were observed during the peaks in GIC. Only during the storm sudden commencement at the beginning of the event were large-scale GIC evident across northern Europe with coherent behaviour. The typical duration of peaks in GIC was also quite short, varying between $2-15 \mathrm{~min}$.
\end{abstract}

Key words. Geomagnetism and paleo-magnetism (geomagnetic induction) - Ionosphere (ionospheric disturbances) Magnetospheric physics (storms and substorms)

Correspondence to: A. Pulkkinen (antti.pulkkinen@fmi.fi)

\section{Introduction}

Rapid geomagnetic variations were first connected with induction phenomena and electric currents flowing in technological systems on the ground in the mid 1800s. In particular, geomagnetically induced currents (GIC) were found to cause disturbances in the operation of telegraph equipment (Barlow, 1849). Since then the number of technological systems having long conductors has increased rapidly, and various networks, such as power transmission systems, buried pipelines, telecommunication cables and railway signalling systems, have been found to be affected by GIC to varying degrees (e.g. Lanzerotti et al., 1999; Boteler et al., 1998).

The basic mechanism producing GIC in conductor systems is quite well understood and several models for a detailed calculation of GIC systems in particular have been developed (e.g. Lehtinen and Pirjola, 1985; Pulkkinen et al., 2001b; Trichtchenko and Boteler, 2001). In general, GIC are driven by rapid variations of ionospheric currents. At high latitudes, where the most intense GIC are experienced, these variations are thought to be related to the intensification of the electrojets during enhanced ionospheric convection conditions and to the development of the substorm current wedge during geomagnetic substorms.

Model calculations of GIC information, based on ionospheric current models, about the Earth's conductivity structure and on the electrical and geometrical characteristics of individual technical systems have been made and corresponding GIC flowing in different parts of conductor systems have been studied (e.g. Viljanen et al., 1999; Favetto and Osella, 1999; Kappenman et al., 2000; Pulkkinen et al., 2001b). Also, studies have been carried out where the geoelectric field is computed directly from the measured ground geomagnetic field (e.g. Viljanen, 1998). With the availability of large geomagnetic databases, these computations have been able to provide statistical estimations of the likelihood of occurrence of GIC in particular systems or regions (e.g. Langlois et al., 1996; Pulkkinen et al., 2000, 2001a).

However, despite numerous studies of GIC, there still ex- 


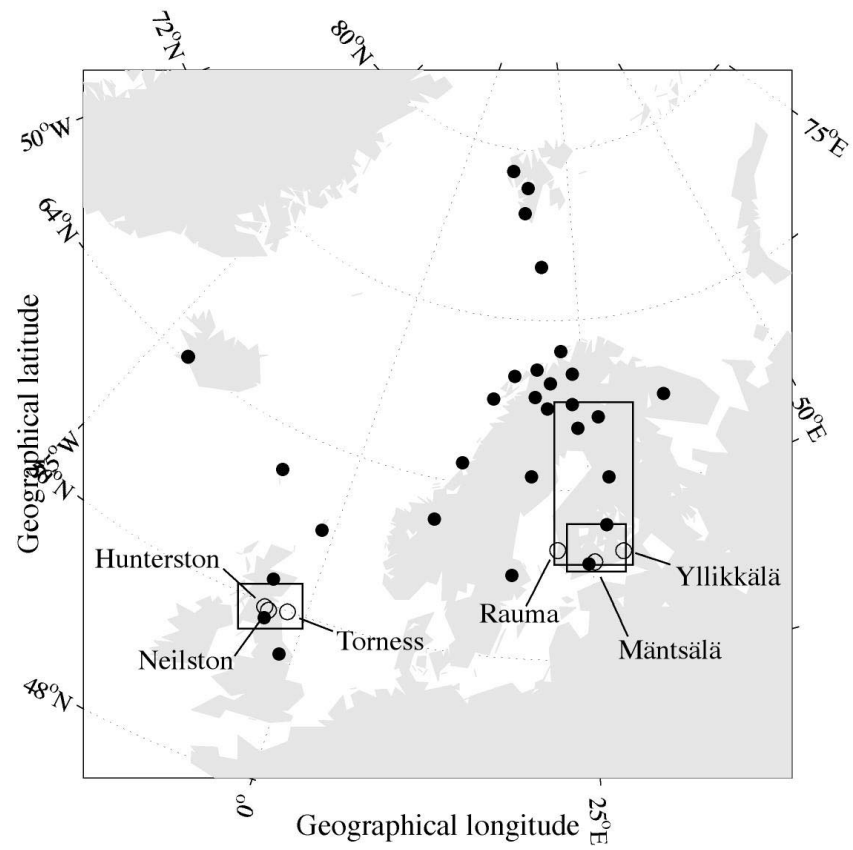

Fig. 1. IMAGE and SAMNET magnetometer array station locations (black dots) and the GIC measurement sites in the Scottish (Hunterston, Neilston, Torness) and Finnish (Rauma, Yllikkälä) power systems and in the Finnish natural gas pipeline (Mäntsälä) (circles). The approximate spatial coverage of the systems is shown with boxes. The westernmost box indicates the Scottish power system, the larger box in the east covers the Finnish power system and the smaller box, the Finnish pipeline.

ists no well-established picture of the detailed structure of the ionospheric currents driving the largest GIC. Some rough estimates of the electrojet intensity and morphology during GIC events have been carried out (e.g. Mäkinen, 1993; Bolduc et al., 1998, 2000; Boteler, 2001), but no rigorous study of realistic ionospheric source currents is available.

The aim of this study is to investigate a single intense geomagnetic storm on 6-7 April 2000. During this storm, large GIC were measured in technological systems, both in Finland and in Great Britain, thus providing GIC data on a relatively large spatial scale. By using these GIC data, together with magnetic measurements from IMAGE and SAMNET magnetometer networks, the ionospheric drivers of the large GIC during the event are identified and described. In parallel with the present investigation is the study by Huttunen et al. (2002), in which the entire Sun-solar wind-magnetosphereionosphere-ground chain was analysed for the same geomagnetic storm. Some of the findings of this study are used to link GIC discussed here to the large-scale solar wind and magnetospheric phenomena.

In Sect. 2, we identify data types and sources and the methods used in the study. Section 3 is devoted to a detailed analysis of the GIC and ionospheric drivers during the April 2000 storm. In Sect. 4 we relate findings in Sect. 3 to larger scale solar wind and magnetospheric dynamics. Finally, in Sect. 5 we briefly summarise our findings and discuss their implications.

\section{Data sources and analysis methods}

The geomagnetic data used in the study were obtained from the IMAGE and SAMNET magnetometer networks operating in the northern part of Europe (Fig. 1). One-minute data were used and a baseline was determined visually for each station before being subtracted from the data to provide the disturbance field. Due to the intensity of the storm, the accuracy of these baselines is not critical.

GIC measurements were obtained from the Scottish and Finnish high-voltage power transmission grids and from the Finnish natural gas pipeline network (Fig. 1). Since we are interested only in general features of the GIC related phenomena, in Fig. 1 we show only the approximate coverage of the networks. For a more detailed study of the behaviour of GIC in specific systems, the detailed structure of the networks would need to be considered.

The power system GIC are found by measuring the current flowing through the neutral line connecting the three phases of the power transformer to the ground. Pipeline GIC measurements are, in turn, carried out by measuring the magnetic field, caused by the current flowing along the pipeline, right above the pipeline (Pulkkinen et al., 2001a). The GIC recordings at the Finnish pipeline commenced in November 1998, and thus, data for over 3.5 years exists to date. All GIC data were produced with a ten-second time resolution.

The geomagnetic data were investigated by the spherical elementary current system (SECS) method (Amm, 1997; Amm and Viljanen, 1999). In this approach, the ground magnetic data are converted to ionospheric equivalent current density patterns.

The separation of the magnetic field into external and internal parts was not carried out prior to computation of equivalent currents. This may result in a slight overestimation of current amplitudes (Tanskanen et al., 2001), but will not have a significant effect on the morphology of current patterns. It should be noted that large spatial gaps in the data result in unreliable estimates of equivalent current patterns (for example, in the region between Svalbard and the Faeroe Islands). However, in areas with relatively high spatial density data, the estimates for equivalent currents can be regarded as reliable (Pulkkinen et al., 2002).

The error of the fit between the measured magnetic field and the magnetic field given by the model at each time step (a measure indicating the ability of the equivalent current model to reproduce the observed fields) is given as a root-meansquare (rms) error, summed over each magnetometer. The median rms during the disturbed period (6 April, 16:40 UT - 7 April, 04:00 UT) is $8 \mathrm{nT}$. This is very small, considering that the amplitudes of geomagnetic variations during the event are several hundreds of nanoteslas.

It should be noted that in general, and excluding some special cases (e.g. Untiedt and Baumjohann, 1993), equivalent currents are not equal to the true 3-D ionospheric current sys- 


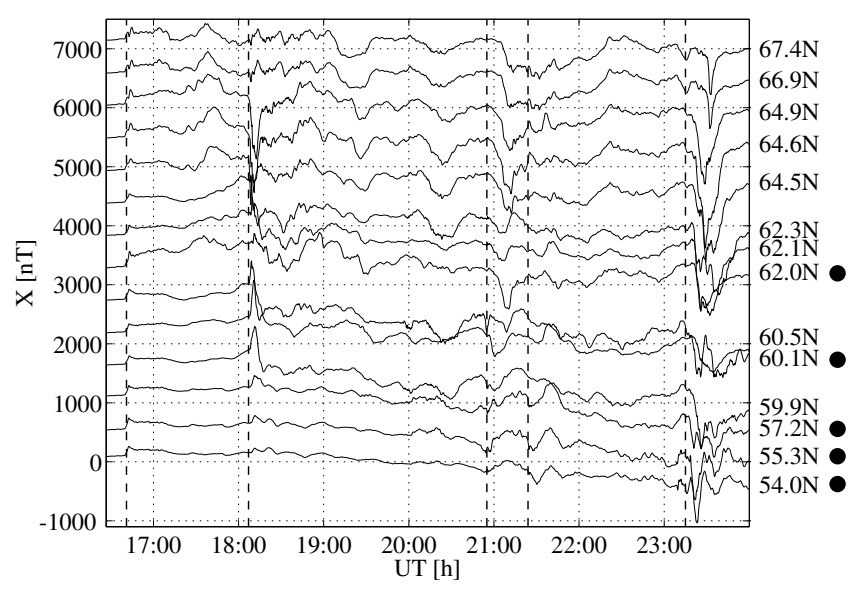

Fig. 2. $X$-component of the ground geomagnetic field on 6 April 2000 at some of the IMAGE and SAMNET stations. Curves for stations are ordered from the south to the north. SAMNET stations are indicated by black dots. Vertical dashed lines indicate the times 16:41, 18:07, 20:55, 21:24 and 23:15 UT, respectively.

tem. In order to solve the true 3-D system, the ionospheric electric field or field-aligned currents over the area need to be supplied (e.g. Amm, 2001). These are not available for the present study. We only have ground magnetic data, which, however, has a relatively good coverage over the region of interest. In addition, equivalent currents are fully sufficient for the purposes of this study.

To examine the characteristics of the electric field at the surface of the Earth, we compute the time derivative of the ground horizontal magnetic field $(d \mathbf{H} / d t, \mathbf{H}=\mathbf{X}+\mathbf{Y})$, to give a reasonable measure of the induction or GIC activity (Viljanen et al., 2001). We then rotate the computed $d \mathbf{H} / d t$ vectors 90 degrees anticlockwise, to be roughly parallel to the large-scale surface electric field, $\mathbf{E}_{\text {sur }}$. Since GIC is closely related to the spatially integrated surface electric field in which small-scale $(\sim 1 \mathrm{~km})$ variations are smoothed out, and since only the basic structure of the induced field is of interest here, $d \mathbf{H} / d t$ is identified with $\mathbf{E}_{\text {sur }}$ below.

\section{Drivers of large GIC during the 6-7 April 2000 geo- magnetic storm}

Before analyzing the drivers of large GIC, we need to define the term "large". The impact of quasi-dc GIC, for example, on power system transformers, depends on the magnitude and duration of the GIC and on the design, loading and set-up of the system (e.g. Kappenman and Albertson, 1990; Lahtinen and Elovaara, 2002). It follows that no general "rule of thumb" on GIC magnitudes can be easily assigned. However, according to statistics by Pulkkinen et al. (2001a), GIC over $5 \mathrm{~A}$ at Mäntsälä station of the Finnish natural gas pipeline can be expected for $100 \mathrm{~min}$ per year, when averaged over several solar cycles. During the 6-7 April 2000 storm, GIC exceeded $5 \mathrm{~A}$ at Mäntsälä for a 25-min period. Thus, we

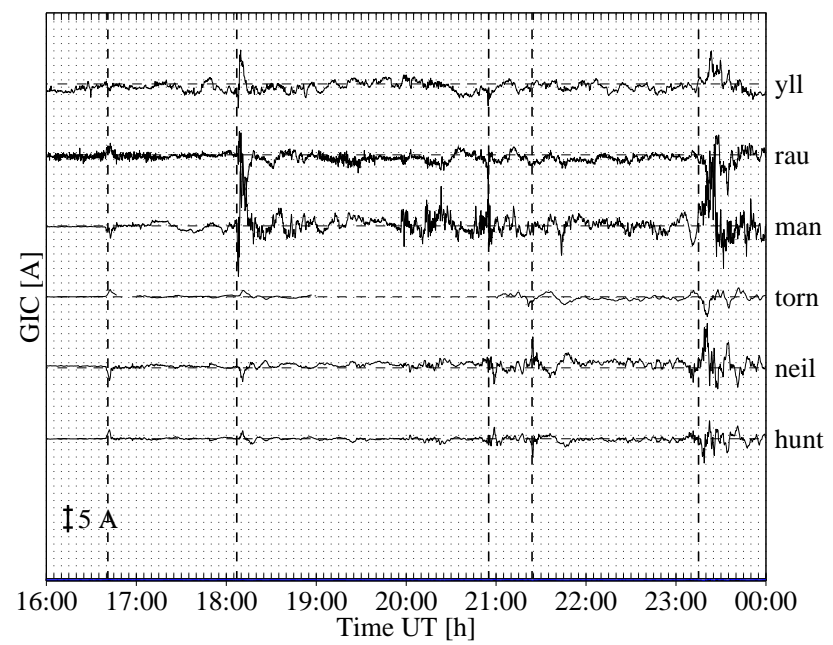

Fig. 3. GIC at the Scottish and Finnish power transformer neutrals and in the Finnish pipeline on 6 April 2000. yll = Yllikkälä, rau $=$ Rauma, man $=$ Mäntsälä, torn $=$ Torness, neil $=$ Neilston, hunt $=$ Hunterston. Vertical dashed lines indicate the times 16:41, 18:07, 20:55, 21:24 and 23:15 UT, respectively.

might anticipate four geomagnetic storms having the activity level of the April 2000 storm per year at Mäntsälä. So we may say that GIC of $5 \mathrm{~A}$ are relatively rare at Mäntsälä and that $5 \mathrm{~A}$ is, therefore, considered our definition of "large". Also note that in the Scottish power grid, GIC exceeding 5 A have been observed only a few times since continuous measurements began in 2000 .

At the onset of the storm, at 16:41 UT, the geomagnetic sudden commencement (SC) produced a global enhancement of the geomagnetic field (Fig. 2) and caused the first large GIC both in the UK and Finland (Fig. 3). The amplitude of GIC is $2-5 \mathrm{~A}$ at all the measurement locations and the enhancement of the GIC lasts about 5 mins. The $\mathbf{E}_{\text {sur }}$ pattern (Fig. 5) shows a coherent west to southwest orientation during the SC (up to 16:40:00-16:41:40 UT). Around 16:42 $\mathbf{E}_{\text {sur }}$ in Fennoscandia adopts a more north-south orientation, but remains westerly over the UK. Subsequently, a pair of regions is established in which $\mathbf{E}_{\text {sur }}$ is driven westward, south of the 60 degrees geomagnetic latitude, and eastwards, north of 60 degrees geomagnetic latitude. This structure persists for only one minute before reversing in sign. Thereafter, there are a number of intervening periods of north-south $\mathbf{E}_{\text {sur }}$. By 16:47 UT, when GIC have already dropped, $\mathbf{E}_{\text {sur }}$ becomes largely irregular over the region. Thus, the first impact is quite coherent across the studied region. This is in accordance with the basic SC model of Araki (1994), in which a global magnetic signature is observed for a few minutes during the passage of the MHD wave produced by the impact of the interplanetary shock. However, the pulse shape usually observed during a SC in the magnetic field in the local afternoon is not clearly present.

At 18:07 UT large enhancements of GIC are observed at the Finnish stations, with the largest value ( $20 \mathrm{~A}$ ) being mea- 


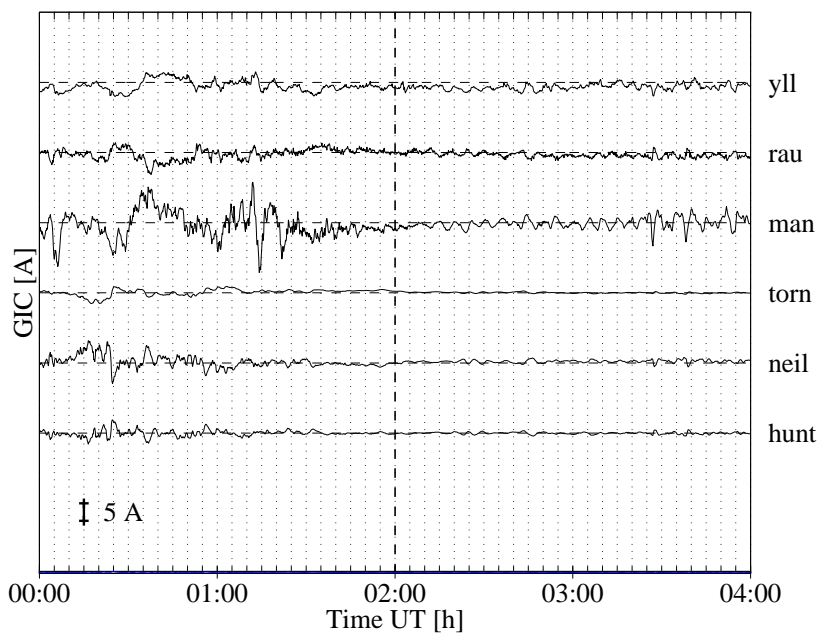

Fig. 4. GIC at the Scottish and Finnish power transformer neutrals and in the Finnish pipeline on 7 April 2000. yll = Yllikkälä, rau $=$ Rauma, $\operatorname{man}=$ Mäntsälä, torn $=$ Torness, neil $=$ Neilston, hunt $=$ Hunterston. The vertical dashed line indicates the time 02:00 UT.

sured at Mäntsälä. These GIC coincide with a substorm onset that causes the intensification of the eastward electrojet and the penetration of the westward electrojet into the Fennoscandian region (Fig. 6). Only a couple of minutes after the intensifications at the Finnish stations, GIC enhancements are also observed in the UK. However, the amplitudes of GIC in the UK are roughly half of those observed in Finland. Presumably this follows from the fact that the eastward electrojet is just north of the British Isles and thus, the largest geomagnetic disturbances caused by this substorm are not seen in the UK. Prior to 18:07 UT, $\mathbf{E}_{\text {sur }}$ shows little coherence across northern Europe. Between 18:07 and 18:09 UT, a localized enhanced $\mathbf{E}_{\text {sur }}$ is observed to develop over Finland and Sweden, between 50 and 60 degrees geomagnetic latitude. One characteristic is that the orientation of the $\mathbf{E}_{s u r}$ pattern changes rapidly. In the UK, $\mathbf{E}_{s u r}$ is consistently westwards over this time interval, turning eastwards after 18:12 UT. By 18:15 UT, $\mathbf{E}_{\text {sur }}$ has returned to being small and irregularly oriented across the whole region. Large Finnish GIC (>5 A) are observed for a period of approximately $6-7 \mathrm{~min}$. The period corresponds roughly to the intensification of the substorm time electrojets.

During the period 19:50-22:00 UT, several GIC enhancements are seen both in the UK and in Finland. Let us concentrate on events that took place at 20:55 UT and 21:24 UT. At 20:55 UT a westward electrojet enhancement was accompanied by a very local current system in the vicinity of the Nurmijärvi Geophysical Observatory. Although some magnetic signatures are seen at other IMAGE stations, $\mathbf{E}_{s u r}$ is several times larger at Nurmijärvi. Thus, the spatial scale of the most intense current system is around $100 \mathrm{~km}$, at most. (Unfortunately, a more detailed study of the structure in the ionosphere is impossible, due to the lack of high spatial density data.) This localized current system produced GIC of 14 A at Mäntsälä. Only about half of this current was mea-

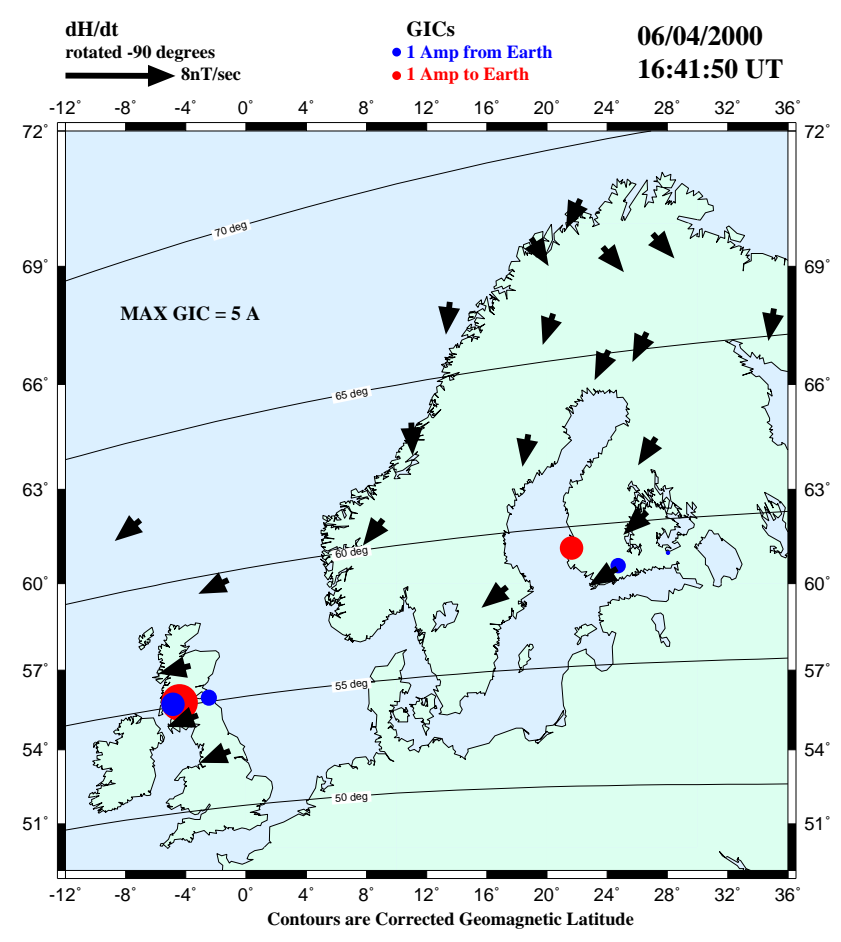

Fig. 5. GIC at the Scottish and Finnish power transformer neutrals and in the Finnish pipeline and the 90 degrees anticlockwise rotated $d \mathbf{H} / d t$ pattern $\left(\mathbf{E}_{s u r}\right.$ ) of the geomagnetic field on the ground on 6 April 2000 at 16:41 UT. Red indicates current toward the east at Mäntälä. See text in Sect. 3 for details.

sured at other Finnish stations, emphasizing the locality of the driver. Scottish measurements were between 3 A -4.5 $\mathrm{A}$ and thus clearly above any background noise level. This suggests that intense current changes in the vicinity of Nurmijärvi may be embedded in some larger scale changes of ionospheric currents. GIC above $5 \mathrm{~A}$ are observed for one minute at Mäntsälä. At 21:24 UT GIC enhancements are observed at the UK stations. This is the only time during the entire storm period when large GIC are observed only in the UK, with no clear enhancement of GIC at the Finnish stations. In terms of equivalent currents, it is the development of the westward current above the UK that drives the GIC (see Fig. 7). The enhancement of currents above the UK is related to the reconfiguration of the westward electrojet above the IMAGE chain. This is seen from Fig. 8, where the difference of equivalent currents at 21:23 and 21:26 UT is shown, and where, apart from a region above the north of the UK, an enhancement of equivalent currents is also observed above southern Finland (at about $60^{\circ} \mathrm{N}$ ). In addition, a change in currents is observed in quite a narrow region in central Fennoscandia (at about $65^{\circ} \mathrm{N}$ ). This is a configuration which itself is quite interesting. A similar small-scale current structure roughly at the same latitude is discussed by Untiedt and Baumjohann (1993, p. 333-334), who noted that such structures are often associated with field-aligned current regions. GIC above $4 \mathrm{~A}$ are observed for about $1-2 \mathrm{~min}$ at the Scottish stations. 


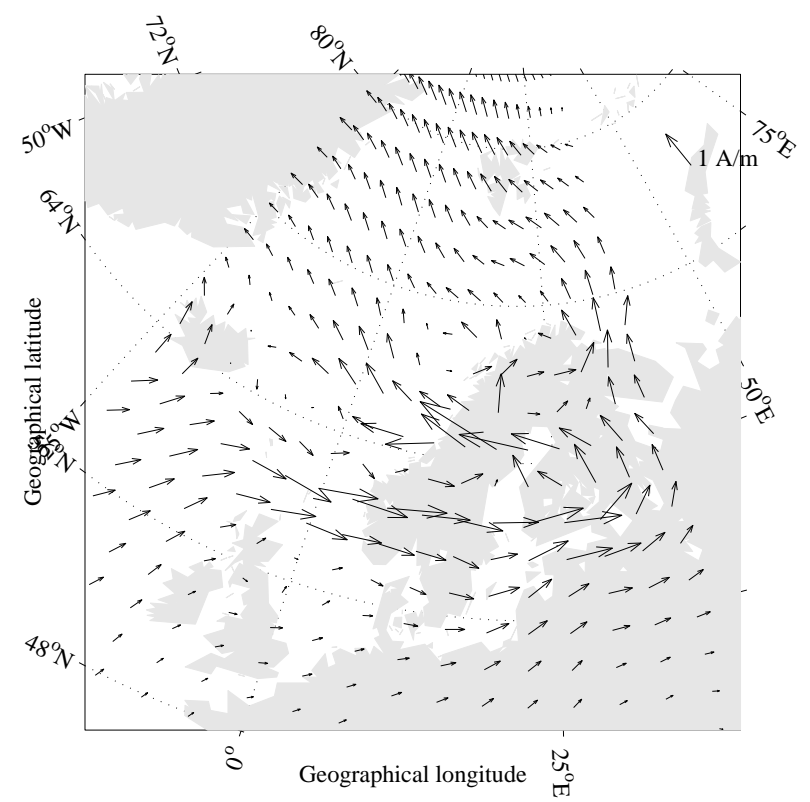

Fig. 6. Ionospheric equivalent currents on 6 April 2000, at 18:09 UT. Rms error of the time step is $16 \mathrm{nT}$ (see text in Sect. 2 for details).

At 23:05 UT the westward electrojet is located over the southern part of the IMAGE network and is also observed in the UK part of SAMNET. Changes in the amplitude of the electrojet at 23:05-23:13 UT (Fig. 9) causes a smooth westward GIC of $5 \mathrm{~A}$ at Mäntsälä. It is quite remarkable that no similar smooth GIC is observed at any other station. This can be explained by looking at the $\mathbf{E}_{s u r}$ pattern for the corresponding period from which we see that $\mathbf{E}_{\text {sur }}$ remains broadly westward only at the vicinity of Mäntsälä, until about 23:12 UT, after which it becomes as variable as in the vicinity of the other GIC measurement sites, with a corresponding decline in current magnitudes. GIC above $2 \mathrm{~A}$ is observed for 2-3 min at Mäntsälä.

At 23:15 UT a large substorm onset takes place. The initial magnetic disturbance at the Earth's surface propagates northward, which is a typical feature of substorms (e.g. Rostoker, 1996). Propagation can be easily seen from Fig. 10, in which the time derivatives of the north component of the magnetic field at some of the SAMNET and IMAGE stations are shown. The time derivative behaves very irregularly and indicates that even during clearly identifiable substorm events, when the intensity of the westward electrojet sharply increases, large contributions to GIC come from the small and rapidly changing currents superimposed on the electrojet. $\mathbf{E}_{\text {sur }}$ patterns (Fig. 11) switch to a more predominantly eastward direction during the intervals of strong GIC occurring across northern Europe. However, rapid orientation swings also remain, particularly over the UK during this time. The largest GIC are observed during the expansion phase of the substorm, with peak values varying between 23 A (Mäntsälä) and 5 A (Torness). After the expansion phase

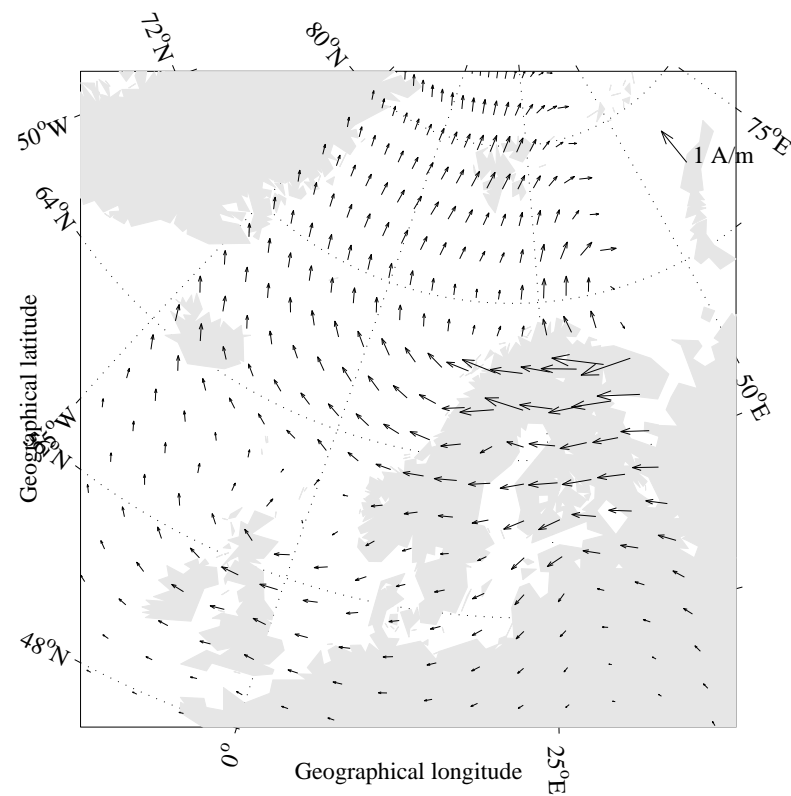

Fig. 7. Ionospheric equivalent currents on 6 April 2000, at 21:24 UT. Rms error of the time step is $15 \mathrm{nT}$ (see text in Sect. 2 for details).

of the substorm (roughly at 23:25 UT) GIC, especially at the Finnish stations, decrease notably. However, oscillations of the decreasing westward electrojet and smaller scale current systems cause large GIC at Finnish and UK stations during the rest of the day.

At the beginning of 7 April, the geomagnetic storm has passed its main phase. Southern Finland and northern UK are under an enhanced westward electrojet. Variations of the electrojet cause large GIC in these areas throughout the period 00:00-01:30 UT (Fig. 4). What is of interest to note is that, according to Huttunen et al. (2002), the only clear substorm of the period is seen at 00:30 UT. However, the effect of this substorm does not appear clearly in GIC measurements. Equally large, or larger GIC are observed during other times between 00:00 and 01:30 UT. The westward electrojet moves a few degrees northward after 01:00 UT, which causes GIC at UK stations to decrease ahead of GIC at Finnish stations. $\mathbf{E}_{\text {sur }}$ is largely eastward over Finland and Scotland for a few minutes following 00:35 UT and again, to a lesser extent, after 00:41 UT. Otherwise, the electric field orientation is strongly variable between east and west in these regions. In the UK a westward $\mathbf{E}_{\text {sur }}$ is established for a few minutes around 01:02 UT, driving currents of a few amps, before turning northwards again. The largest GIC of the period are measured at Mäntsälä, where several peaks exceeding the $10 \mathrm{~A}$ are observed. Although oscillations are not as rapid as during the beginning of the storm, the durations of large GIC are less than $15 \mathrm{~min}$.

During the period 02:00-04:30 UT, coincident with the recovery phase of the storm, geomagnetic pulsations drive GIC at all stations. A rough estimate for the period of the pulsa- 


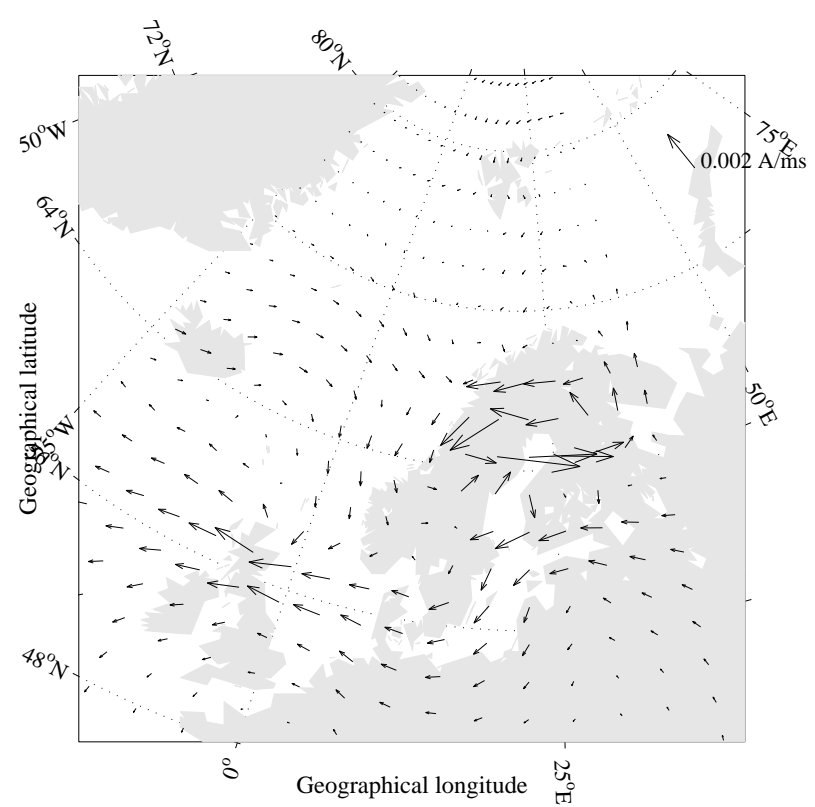

Fig. 8. Difference of ionospheric equivalent currents on 6 April 2000. Currents at 21:23 are subtracted from currents at 21:26 UT. Rms error at the time step 21:23 UT is $12 \mathrm{nT}$; rms error at the time step 21:26 UT is $14 \mathrm{nT}$ (see text in Sect. 2 for details). See also Fig. 7.

tions is $5-8 \mathrm{~min}$, which makes them fall into the Pc5 category. Pc5 pulsations are thought to be produced by solar wind driven field line resonances that occur at higher latitudes (e.g. Chisham and Orr, 1997). The amplitudes of the pulsations decrease towards the south and thus, they create larger GIC at the Finnish stations, with the largest amplitudes $(2.5 \mathrm{~A})$ located again at Mäntsälä. Despite the apparent coherency of the magnetic signal caused by pulsations, there are no large-scale structures in $\mathbf{E}_{\text {sur }}$ that behave coherently across the whole region at any point between 02:00 and 04:00 UT. Local structures are seen (for example, on the scale of the UK, or of Fennoscandia, north of 60 degrees geomagnetic), with a tendency for neighboring $\mathbf{E}_{s u r}$ vectors to change from west to east or conversely regularly over the time frame. Reversals in the direction of $\mathbf{E}_{\text {sur }}$ can take 510 min to accomplish and $\mathbf{E}_{\text {sur }}$ remains in the new orientation for a similar time. At the very end of the storm event, an increase in GIC at all stations is observed between 03:2503:55 UT, perhaps due to an increase in the amplitude of pulsations, or due to the appearance of some other current system superimposed on currents associated with the pulsations. The largest amplitude GIC (6.5 A) is observed at Mäntsälä.

\section{Discussion}

We have described in detail characteristics of large GIC and corresponding ionospheric equivalent current systems and ground electric fields during the 6-7 April 2001 magnetic storm. Below, their relation to larger scale solar wind

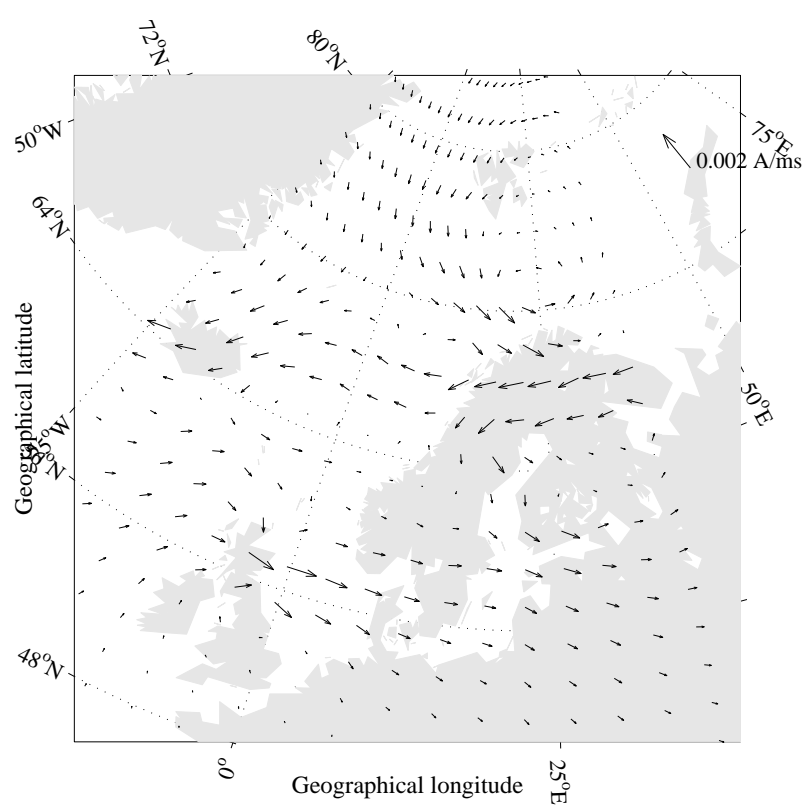

Fig. 9. Difference of ionospheric equivalent currents on 6 April 2000. Currents at 23:05 are subtracted from currents at 23:13 UT. Rms error at the time step 23:05 UT is $15 \mathrm{nT}$; rms error at the time step 23:13 UT is $8 \mathrm{nT}$ (see text in Sect. 2 for details).

and magnetospheric dynamics is discussed. The discussion is based mainly on the results by Huttunen et al. (2002), who investigated this storm from the viewpoint of the Sunsolar wind-magnetosphere-ionosphere-ground chain. Some of their key results, which have relevance for our study, are summarized below.

On 4 April 2000, a coronal mass ejection (CME) was observed to leave the Sun. Although the ejecta was not believed to be directly earthward, the sheath region between the shock front and the driver gas hit the Earth's magnetosphere, causing the largest geomagnetic storm of 2000, as measured by the low-latitude Dst index. The shock impact was observed at 16:40 UT at geostationary orbit on the dayside. Solar wind plasma conditions, namely strongly negative and fluctuating IMF and high dynamic pressure in the sheath region behind the shock produced strongly driven magnetospheric activity. Several ionospheric activations occurred all around the auroral oval during the storm. Four of these activations, identified from the IMAGE data, showed substorm-like behavior (18:05, 20:13, 23:15, 00:30 UT, poleward and westward expansion of the nightside ionospheric current system, accompanied by particle injections observed at geostationary orbit in the midnight region) and were thus related to the release of the energy stored in the magnetotail. The cause of the increased energy storage during the storm was the strong driving caused by the negative $B_{z}$ and the high pressure of the solar wind. Four other activations (17:08, 19:10, 20:55, 22:16 UT) were related to the expansion of the ionospheric current system but clear particle injections were missing. It was suggested by Huttunen et al. (2002) that the first set of 


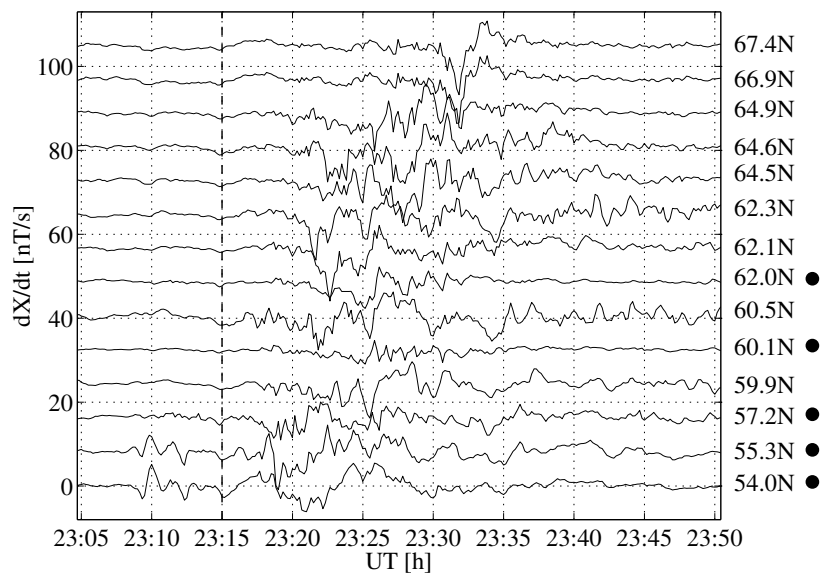

Fig. 10. The time derivatives of the $X$-component of the ground geomagnetic field on 6 April 2000 at some of the IMAGE and SAMNET stations. Curves for stations are ordered from the south to the north. SAMNET stations are indicated by black dots. Vertical dashed line indicates the time 23:15 UT.

activations, especially the last two substorms, was associated with both increased energy input from the solar wind and dynamic reconfigurations in the magnetotail, whereas the latter activations were possibly direct responses to the solar wind driving.

The largest GIC of the storm period occurred at 18:07 and 23:15 UT (6 April) and were associated with substorms. Both substorms caused large, but incoherent $\mathbf{E}_{\text {sur }}$ at the area of northern Europe. Most notably, during the intensification at 23:15 UT, the expansion of substorm related ionospheric currents from lower latitudes towards the north caused a sweep of the ionospheric region. This caused large GIC practically throughout northern Europe.

Localized small-scale features were one of the main characters of the storm period. In general, it was seen that although the presence of the auroral electrojet favored large GIC, the actual reason for GIC were small-scale features in the ionosphere. In other words, usually $|\boldsymbol{X}| \gg|\boldsymbol{Y}|$ but $|d \boldsymbol{X} / d t| \approx|d \boldsymbol{Y} / d t|$, which can be explained only by smallscale variations in the source currents. The duration of individual GIC spikes was short (a few minutes) and $\mathbf{E}_{\text {sur }}$ patterns were changing rapidly and were inhomogeneous as well, which is in accordance with the results by Viljanen et al. (2001), who studied the statistical behavior of the time derivatives of the ground magnetic field. From the substorm's point of view, this type of behavior is quite expected, taking into account the turbulent nature of the phenomenon (e.g. Klimas et al., 2000).

Strong driving of the magnetospheric activity causes the auroral oval to expand towards lower latitudes. At such times, variations in ionospheric currents (electrojets) can cause large GIC at latitudes where GIC is not typically experienced. An example can be seen at 21:24 UT, when the electrojet above the UK embodies variations in currents that cause large GIC at Scottish stations, while Finnish stations

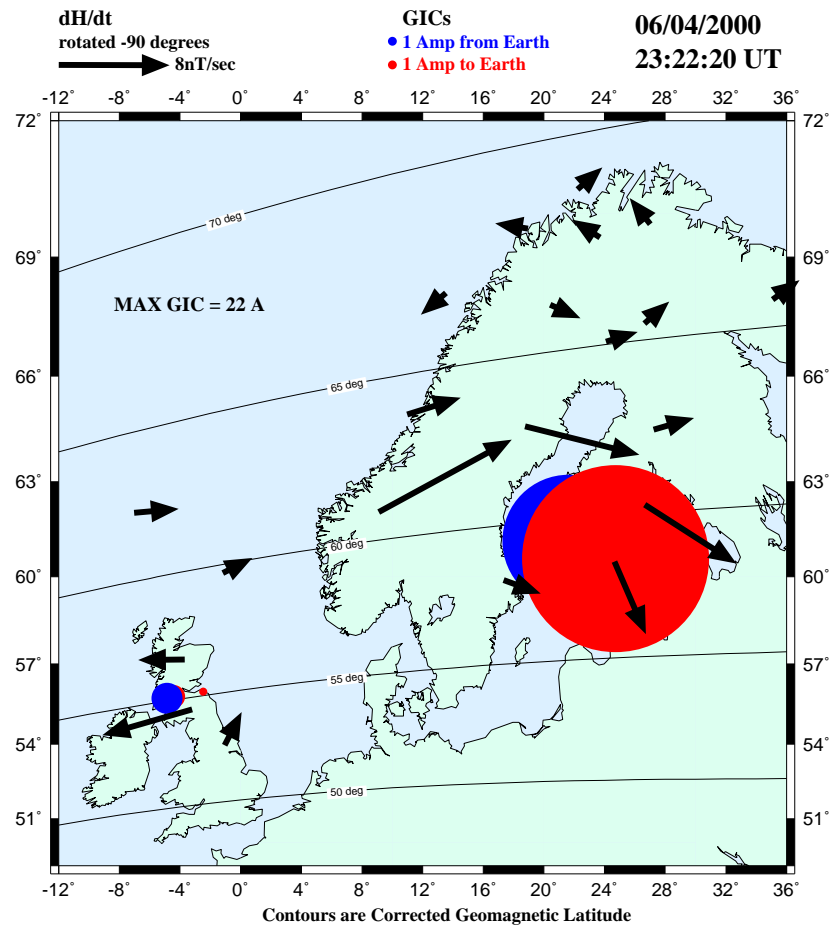

Fig. 11. GIC at the Scottish and Finnish power transformer neutrals and in the Finnish pipeline and the 90 degrees anticlockwise rotated $d \mathbf{H} / d t$ pattern $\left(\mathbf{E}_{s u r}\right)$ of the geomagnetic field on ground on 6 April 2000 at 23:22 UT. Red indicates current toward the east at Mäntsälä. See text in Sect. 3 for details.

do not observe clear GIC enhancements.

Not all large GIC were associated with substorms. For example, at 20:55 UT substorm onset type intensification was observed at magnetometer stations. However, as was explained above, due to the missing signature of particle injections at geostationary orbit, its morphology is distinct from the classical substorm event. Thus, corresponding large localized GIC at Mäntsälä cannot be accounted for by substorms, but perhaps by some finer structure behavior of the magnetosphere that was directly driven by the solar wind, as suggested by Huttunen et al. (2002). In addition, the geomagnetic sudden commencement (SC) caused by the intense shock related pressure pulse in the solar wind was seen to cause GIC on a large spatial scale. Shock fronts are often associated with intense geomagnetic storms (e.g. Tsurutani and Gonzalez, 1997) and thus, SCs are one important type of reason for storm related GIC. SC preceding the magnetic storm on 24 March 1991 caused one of the largest ever observed GIC (175 A) in the Finnish power system (Viljanen and Pirjola, 1994). On 7 April solar wind directly driven geomagnetic pulsations in the local morning region caused a sequence of large GIC. According to Viljanen et al. (2001), local morning is one of the time sectors where large ground magnetic variations (and large $\mathbf{E}_{\text {sur }}$ ) are typically observed. These variations can possibly be partly explained by pulsations. It should be noted that although the amplitude of the pulsation related GIC were smaller than during substorms, 


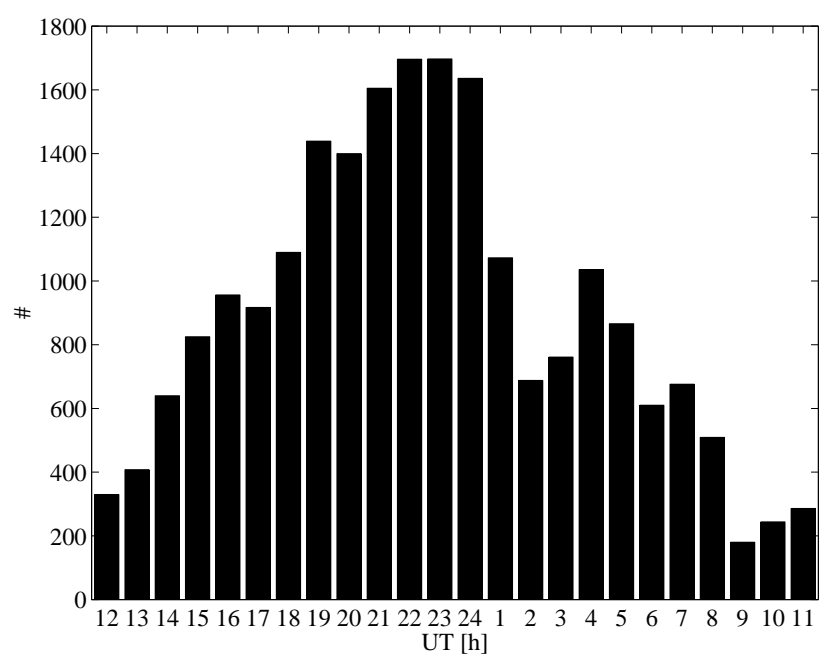

Fig. 12. Statistical occurrence of GIC above $2.5 \mathrm{~A}$ in the Finnish pipeline. Counts computed for each UT sector correspond to the number of measured 10-s values of GIC above 2.5 A. Statistics are derived using data from November 1998 to May 2002.

the long duration (approx. 02:00-04:30 UT) of the event may give rise, for example, to cumulative corrosion effects experienced by buried pipelines (e.g. Martin, 1993).

To investigate how the GIC driver dynamics discussed above relate to the statistical picture, we computed the statistical occurrence of GIC above $2.5 \mathrm{~A}$ (GIC above 5 A has occurred too rarely for statistical considerations) in the Finnish pipeline. Statistics were computed for each UT sector, revealing the times when large GIC are typically observed. Finnish local time is UT +2 hours. The result is an updated version of the data of Viljanen et al. (2001), where now data from November 1998 to May 2002 are used. In Fig. 12 it can be seen that two clearly distinguishable peaks are present: one in the vicinity of the local midnight, another in the local morning sector. It is known that the most dramatic changes in ionospheric currents during substorms happen in the vicinity of local midnight, and thus, one is tempted to propose that substorms are also in a statistical sense the most important cause for large GIC in the auroral regions. Correspondingly, it is known that many classes of auroral region ULF waves, like Pc5s, are favored at the local morning region (e.g. Vennerstrøm, 1999), which was also observed during the April 2000 storm. Thus, it may be that pulsations are the second largest category of cause for large GIC.

Summarizing, clearly identifiable ionospheric drivers of large GIC during the 6-7 April 2000 geomagnetic storm were related to (1) the SSC at the beginning of the storm, (2) pulsations at the end of the storm, (3) substorm onsets during the storm. In addition, various small-scale, although not easily classifiable, features were observed to cause large GIC. Statistical considerations suggested that substorms and pulsations are the most typical cause of large GIC at the auroral region.

\section{Conclusions}

Although most of the very largest GIC during the April 2000 storm were clearly related to substorm intensifications, a conclusion supported also by the statistics, there were no common characteristics in the substorm behavior that could be associated with all the large GIC. For example, both very localized ionospheric current structures and relatively largescale, propagating structures were observed during individual peak GIC. Only during the sudden commencement at the beginning of the event where there large-scale coherent GIC and surface electric fields across northern Europe. A typical duration of the peak GIC was generally quite short, typically varying between $2-15 \mathrm{~min}$.

In light of the present study, one key issue, related to the possibility of GIC forecasting in the auroral regions, is the predictability of substorms. For this, the time of onset and the location of ionospheric current intensifications need to be known. If these characteristics are given with a sufficient accuracy, regional GIC warnings may be given with a reliability that might be acceptable to potential space weather customers. However, it is clear that for the moment accurate GIC predictions, where the behavior of GIC at individual sites is computed, are not possible with the present knowledge. The dynamics of ionospheric currents have been shown to be too complex for existing predictive methods to cope with.

Although individual case studies, such as those presented here, provide a good basis for understanding basic processes causing large GIC, a more general classification, using a larger database, is required. This will allow a more quantitative statement of the relative importance of the different ionospheric events producing large ground effects. Such a classification will be one of our goals in future GIC studies. As a start, the more recent 15-16 July 2000 magnetic storm will be of special interest, due to the extremely large GIC observed in Europe.

Acknowledgements. The work of Antti Pulkkinen was supported by the Academy of Finland. The IMAGE magnetometer array is an international project headed by the Finnish Meteorological Institute (http://www.geo.fmi.fi/image). The authors thank Prof I. R. Mann and Dr D. K. Milling for the SAMNET data. SAMNET is a PPARC National Facility deployed and operated by the University of York. Helpful discussions with Drs. Ari Viljanen, Risto Pirjola and Toby Clark are acknowledged. Fingrid Oyj, Scottish Power and Gasum Oy are thanked for their data and support. This paper is published with the permission of the Executive Director, British Geological Survey (NERC).

Topical Editor M. Lester thanks H. Lühr and A. Yahnin for their help in evaluating this paper.

\section{References}

Amm, O.: Ionospheric elementary current systems in spherical coordinates and their application, J. Geomagn. Geoelectr., 49, 947, 1997. 
Amm, O.: The elementary current method for calculating ionospheric current systems from multisatellite and ground magnetometer data, J. Geophys. Res., 106, 24 843, 2001.

Amm, O. and Viljanen, A.: Ionospheric disturbance magnetic field continuation from the ground to ionosphere using spherical elementary current systems, Earth, Planets and Space, 51, 431, 1999.

Araki, T.: A physical model of the geomagnetic sudden commencement, Solar Wind Sources of Magnetospheric Ultra-LowFrequency Waves, Geophys. Monogr. Ser., 81, (Eds) Engebretson, M. J., Takahashi, K., and Scholer, M., p. 183, AGU, Washington, D. C., 1994.

Barlow, W. H.: On the spontaneous electrical currents observed in wires of the electric telegraph, Royal Society of London, Philosophical Transactions, 139, 61, 1849.

Bolduc, L., Langlois, P., Boteler, D., and Pirjola, R.: A Study of Geoelectromagnetic Disturbances in Québec, 1. General Results, IEEE Trans. Power Delivery, 13, 1251, 1998.

Bolduc, L., Langlois, P., Boteler, D., and Pirjola, R.: A Study of Geoelectromagnetic Disturbances in Québec, 2. Detailed Analysis of a Large Event, IEEE Trans. Power Delivery, 15, 272, 2000.

Boteler, D.: Space Weather Effects on Power Systems, Space Weather, AGU Geophysical Monograph 125, 347, 2001.

Boteler, D. H., Pirjola, R. J., and Nevanlinna, H.: The Effects of Geomagnetic Disturbances on Electrical Systems at the Earth's Surface, Adv. Space Res., 22, 17, 1998

Chisham, G. and Orr, D.: A statistical study of the local time asymmetry of Pc5 ULF wave characteristics observed at midlatitudes by SAMNET, J. Geophys. Res., 102, 24 339, 1997.

Favetto, A. and Osella, A.: Numerical Simulation of Currents Induced by Geomagnetic Storms on Buried Pipelines: An application to the Tierra del Fuego, Argentina, Gas Transmission Route, IEEE T. Geosci. Remote, 37, 614, 1999.

Huttunen, K. E. J., Koskinen, H. E. J, Pulkkinen, T. I., Pulkkinen, A., and Palmroth, M.: April 2000 storm: Solar wind driver and magnetospheric response, J. Geophys. Res., 107, doi: 10.1029/2002JA0099154, 2002.

Kappenman, J. G. and Albertson, V. D.: Bracing for the Geomagnetic Storms, IEEE Spectrum, 27, 1990.

Kappenman, J. G., Radasky, W. A., Gilbert, J. L., and Erinmez, I. A.: Advanced geomagnetic storm forecasting: a risk management tool for electric power system operations, IEEE T. Plasma Sci., 28, 2114, 2000.

Klimas, A. J., Valvidia, J. A., Vassiliadis, D., Baker, D. N., Hesse, M., and Takalo, J.: Self-organized criticality in the substorm phenomenon and its relation to localized reconnection in the magnetospheric plasma sheet, J. Geophys. Res., 105, 18 765, 2000.

Lahtinen, M. and Elovaara, J.: GIC Occurrences and GIC Test for 400 kV System Transformer, IEEE Trans. Power Delivery, 17, 555, 2002

Langlois, P., Bolduc, L., and Chouteau, M. C.: Probability of Occurrence of Geomagnetic Storms Based on a Study of the Distribution of the Electric field Amplitudes Measured in Abitibi, Québec, in 1993-94, J. Geomagn. Geoelectr., 48, 1033, 1996.

Lanzerotti, L., Thomson, D., and Maclennan, C.: Engineering Issues in Space Weather, Modern Radio Science 1999, Published for the International Union of Radio Science by Oxford University Press, 1999.

Lehtinen, M. and Pirjola, R.: Currents produced in earthed conductor networks by geomagnetically-induced electric fields, Ann Geophysicae, 3, 4, 479, 1985.

Martin, B. A.: Telluric Effects on a Buried Pipeline, Corrosion, 49, 343, 1993.

Mäkinen, T.: Geomagnetically Induced Currents in the Finnish Power System, Geophysical Publications, No. 32, Finnish Meteorological Institute, p. 101, 1993.

Pulkkinen, A., Viljanen, A., Pirjola, R., and BEAR Working Group: Large Geomagnetically Induced Currents in the Finnish HighVoltage Power System, Finnish Meteorological Institute, Reports, 2000:2, p. 99, 2000.

Pulkkinen, A., Viljanen, A., Pajunpää, K., and Pirjola, R.: Recordings and occurrence of geomagnetically induced currents in the Finnish natural gas pipeline network, J. Appl. Geophys., 48, 219, 2001a.

Pulkkinen, A., Pirjola, R., Boteler, D., Viljanen, A., and Yegorov, I.: Modelling of space weather effects on pipelines, J. Appl. Geophys., 48, 233, 2001b.

Pulkkinen, A., Amm, O., Viljanen, A., and BEAR Working Group: Ionospheric equivalent current distributions determined with the method of elementary current systems, J. Geophys. Res., in press, 2002.

Rostoker, G., Phenomenology and physics of magnetospheric substorms, J. Geophys. Res., 101, 12 955, 1996.

Tanskanen, E. I., Viljanen, A., Pulkkinen, T. I., Pirjola, R., Häkkinen, L., Pulkkinen, A., and Amm, O.: At substorm onset, $40 \%$ of AL comes from underground, J. Geophys. Res., 106, $13119,2001$.

Trichtchenko, L. and Boteler, D. H.: Specification of geomagnetically induced electric fields and currents in pipelines J. Geophys. Res., $106,21039,2001$.

Tsurutani, B. T. and Gonzalez, W. D.: The interplanetary causes of magnetic storms: A review, Magnetic Storms, AGU Geophysical Monograph 98, 77, 1997.

Untiedt, J. and Baumjohann, W.: Studies of polar current systems using the IMS Scandinavian magnetometer array, Space Sci. Rev., 63, 245, 1993.

Vennerstrøm, S.: Dayside magnetic ULF power at high latitudes: A possible long-term proxy for the solar wind velocity?, J. Geophys. Res., 104, 10 145, 1999.

Viljanen, A.: Relation of Geomagnetically Induced Currents and Local Geomagnetic Variations, IEEE Trans. Power Delivery., 4, 1285, 1998.

Viljanen, A. and Pirjola, R.: Geomagnetically induced currents in the Finnish high-voltage power system, Surv. Geophys., 15, 383, 1994.

Viljanen, A., Amm, O., and Pirjola, R.: Modeling geomagnetically induced currents during different ionospheric situations, J. Geophys. Res., 104, 28 059, 1999.

Viljanen, A., Nevanlinna, H., Pajunpää, K., and Pulkkinen, A.: Time derivative of the horizontal geomagnetic field as an activity indicator, Ann. Geophysicae, 19, 1107, 2001. 